\title{
Leserservice 2020 - Zahlen und Fakten
}

Die Corona-Pandemie hat die an dieser Stelle traditionell veröffentlichte Benutzungsstatistik mit Blick auf das Jahr 2020 gehörig durcheinander gebracht. Nach nur 229 Öffnungstagen und einem wegen gesetzlicher Hygienebestimmungen stark reduziertem Arbeitsplatzangebot sind die meisten Werte nicht mit denen der Vorjahre vergleichbar. Andererseits wurden durch die Pandemiesituation neue Services zur ortsunabhängigen Nutzung der Bibliothek entwickelt, für die es wiederum in der klassischen Benutzungsstatistik keine Kategorien gibt und auf diese im Folgenden näher eingegangen werden soll:

Während des ersten mehrwöchigen Lockdowns ab dem 17. März waren Bibliotheken deutschlandweit für den Publikumsverkehr geschlossen und das Bestellwesen inkl. Fernleihe kam vielerorts komplett zum Erliegen. Die WLB verschickte zwar durchgehend ihre Leihgaben im Rahmen der aktiven Fernleihe, jedoch war das Ausleihsystem bis zum 7. Mai abgestellt, das Mahnwesen ausgesetzt und entliehene Bücher sowie die Laufdauer von Benutzerausweisen wurden automatisch verlängert. Neue Bibliotheksausweise bewilligte die Leihstelle per E-Mail wie auch Medienbestellungen, die man anfangs per Post an die Benutzer schickte, bevor am 20. April eine aktualisierte Corona-Verordnung die kontaktlose Abholung und Rückgabe ermöglichte.

Parallel dazu lizenzierte die WLB verstärkt E-Books und Datenbanken für den Fernzugriff. Ein Blick auf die Statistik zeigt, dass die Nutzung der extern verfügbaren digitalen Medien um 38,6 \% gestiegen ist. Dem gegenüber stehen rückläufige Entleihungen und eine vergleichsweise hohe Anzahl an Verlängerungen von ausgeliehenen Medien. Wie schon erwähnt, hatten die Benutzer der WLB im ersten Lockdown von Beginn an die Möglichkeit, sich Medien, aber auch Kopien aus Büchern oder Zeitschriften per Post zuschicken zu lassen. Der Buch- und Kopienversand, der in Anbetracht der andauernden Infektionslage auch während der moderierten Teilöffnung ab Mai beibehalten wurde, nahm beim zweiten Bibliothekslockdown ab 16. Dezember ordentlich Fahrt auf: In den Tagen vor
Weihnachten gingen bis zu 41 Pakete am Tag auf die Reise, und auch mit Kopienversand konnten noch viele dringende Benutzeranliegen erfüllt werden. Zusammen mit dem telefonischen Beratungsangebot gelang es trotz Schließung der Bibliothek, die Literatur- und Informationsversorgung ein gutes Stück weit aufrechtzuerhalten.

Wie dringend die Bibliothek als Lern- und Arbeitsort benötigt wird, zeigte sich nach der Freigabe der ersten Arbeitsplätze im Hauptlesesaal am 20. April: Anfangs waren es nur 35 Arbeitsplätze und diese Studierenden und Angehörigen der Hochschulen vorbehalten. Ab dem 11. Mai - durch Hinzunahme zusätzlicher Flächen auf der Infoebene - immerhin schon 65 und mit Eröffnung des Erweiterungsbaus am 5. Oktober schließlich 193 Arbeitsplätze, die unter den strengen COVID-19-Bedingungen angeboten werden konnten. Ein Blick in das Reservierungssystem WLB-Riserva belegt die starke Nachfrage nach Arbeitsplätzen mit zuletzt im Dezember durchschnittlich 343 Buchungen am Tag und meistens einer hohen Reservierungsdauer von mehreren Stunden - und das, obwohl ab November auch am Platz permanent eine MundNasenbedeckung getragen werden musste.

Paradoxerweise erhöhte sich ausgerechnet im Corona-Jahr trotz der zahlreichen Schließtage und des reduzierten Arbeitsplatzangebots die Anzahl der aktiven Benutzer erheblich: So schlagen nicht so sehr die Neuanmeldungen, sondern vielmehr die große Anzahl an Gültigkeitsverlängerungen von bestehenden Bibliotheksausweisen in der Statistik stark zu Buche. Wie kommt es, dass ausgerechnet während Corona so viele Benutzer die WLB wiederentdeckt haben? Die Nachfrage nach elektronischen Informationsangeboten ist durch Pandemie und Lockdown rasant angestiegen. Abgelaufene Ausweise wiederum konnten unbürokratisch per E-Mail verlängert werden und ermöglichten ihrem Besitzer sofortigen Zugriff auf umfangreiche digitale Ressourcen im Fernzugriff. Doch auch wer Printmedien benötigte - im Übrigen auch aus dem Freihand- und Präsenzbestand -, konnte sich diese dank Buch- und Kopienversand kostengüns- 
tig zuschicken lassen. Last but not least mussten Arbeitsplätze ab Wiedereröffnung der Bibliothek obligatorisch reserviert werden und dies geht nur mit einem aktiven Bibliotheksausweis. Wer vor Corona die Bibliothek nur als Lernort genutzt hatte, konnte dies theoretisch auch ohne Bibliotheksausweis tun. Durch die Reservierungspflicht wird die Vorort-Nutzung nun künftig adäquater in der Statistik abgebildet.

Birgit Oberhausen

\begin{tabular}{|l|r|r|}
\hline & $\mathbf{2 0 1 9}$ & $\mathbf{2 0 2 0}$ \\
\hline Allgemeine Angaben zur Benutzung & & \\
Aktive Benutzer & 25.220 & 42.644 \\
Gültige Bibliotheksausweise & 35.327 & 53.451 \\
Entleihungen & 1.230 .109 & 988.144 \\
Verlängerungen & 790.310 & 704.226 \\
Auskunftsanfragen Info & 17.248 & 20.636 \\
Aktive Fernleihe & 72.087 & 64.186 \\
Passive Fernleihe & 20.676 & 15.205 \\
Besucher Hauptlesesaal bzw. Arbeitsplätze EB & 256.200 & 57.196 \\
Besucher Sonderlesesaal & 9.797 & 4.009 \\
Zugriffe auf Digitale Medien & & \\
OPAC + Portal & 2.052 .211 & 1.913 .174 \\
Extern nutzbare E-Ressourcen (HAN) & 273.017 & 378.415 \\
Digitale Sammlungen WLB & 1.006 .083 & 1.274 .536 \\
\hline
\end{tabular}

